ORIENTAL JOURNAL OF
ISSN: 0974-6471
August 2016,
COMPUTER SCIENCE \& TECHNOLOGY

\title{
Development of Online Student Course Registration System
}

\author{
RATTAN SINGH, RAVINDER SINGH*, \\ HARPREET KAUR AND O. P. GUPTA \\ Punjab Agricultural University, Ludhiana, Punjab, India. \\ http://dx.doi.org/10.13005/ojcst/9.02.02
}

(Received: June 16, 2016; Accepted: July 20, 2016)

\begin{abstract}
With the advent of Information Technology in the last decade, the major focus has shifted from manual systems to computerized systems. Various systems viz. railway reservation, hospital management etc. involving manual work have been automated efficiently. Student course registration process in colleges involve filling registration forms manually, getting it signed by respective subject teachers, and then getting the documents acknowledged fromthe concerned Advisors, College Deans and Accounts Officers respectively. Finally the registration forms are submitted in the Administrative Branch. As is evident, this process is very laborious and time consuming. An Online Student Course Registration System has been developed to simplify the current manual procedure. This system has been developed using PHP, jQuery, Apache and MySQL. The front-end is designed using PHP with excerpts of code written using jQuery and back-end is designed and managed through MySQL. This system software is more secured, user-friendly and less time-consuming.
\end{abstract}

Keywords: PHP, MySQL, jQuery, Apache, Database, Database Management System, Front-end, Back-end.

\section{INTRODUCTION}

System may be defined as a layered structure that depicts how programs involved would interrelate and communicate. In computers, System may also include actual programs, programming interfaces and tools for managing the larger system. The term system may be used differently in different contexts, but more or less the concept remains the same. Online student course registration system combines multiple systems to construct a combined framework. This framework consists of multiple modules, which further contain different systems along with the implementation of their defined constraints.

Basically, systems are implemented for facilitating complex manual processes and that is exactly what we are trying to achieve. System is implemented as per user requirement such as a manufacturing concern may install a plant for easing out manual processes. We have sought help from computer programming for automation of manual registration system. With the introduction 
of computers, every aspect of our lives has been revolutionized. When used judiciously, computers can help us save time, secure our personal information, access the required information whenever and wherever required. Keeping all these positive points in mind, we have developed an Online Student Course Registration System for easily managing the semester registration process for the student in an institution. Ours is an advisory based system. In state agricultural universities the course allocation is advisory based and more complicated. The courses are assigned according to the skill set and industry requirements. Hence, in current scenario, automated system is required for course registration of students.

\section{Objectives}

The current research aims at reducing the workload all the entitiesinvolved in the registration procedure for the students. The current manual system faces different challenges as to maintaining data of each student manually. Hard copy registers are maintained currently to verify student details.
From students' point of view, they have to fill the forms manually and then get them verified from concerned officials, which is a very time consuming process. The objectives of this proposed web application system are:

- To computerize student and faculty database.

- To maintain data consistency and integrity.

- Automate the registration process without any physical human interaction

- Making the registration process accessible anywhere to the student.

- Allowing faculty to acknowledge registration requests from anywhere.

With the requirement of registration process for every semester, it becomes all the more important to simplify a process which is highly repetitive. The achievement of the above objectives can help the institution in managing the resources efficiently. The automated process will lead to time saving and eradication of common errors.

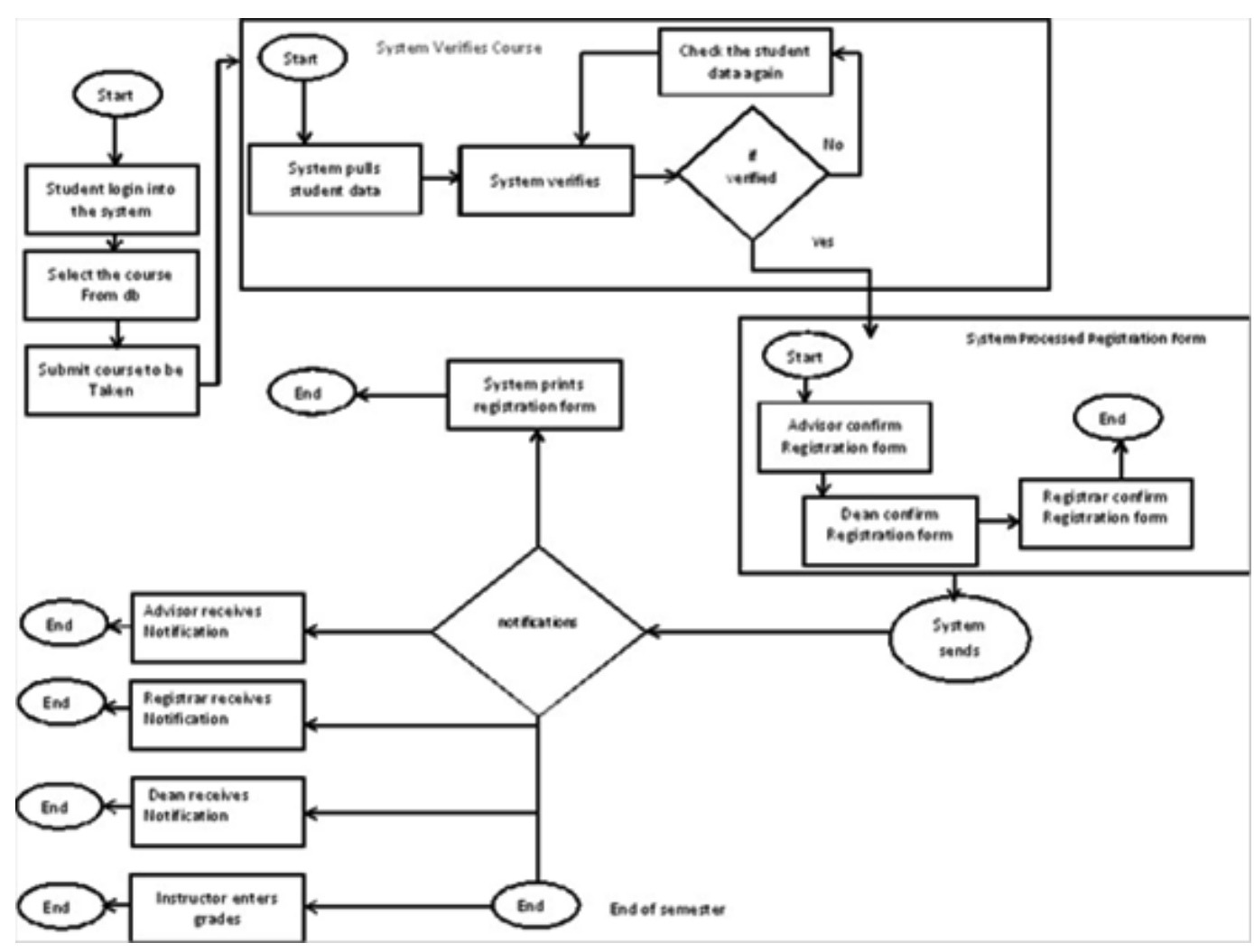

Fig. 1: System Flow of Student Registration 


\section{MATERIAL AND METHODS}

\section{Present System}

The existing system involves the student filling five identical forms called yellow forms which contain student'spersonal details as well as the courses he/she has to register during the next semester. After filling those forms, the student gets them signed by the respective subject teachers and the Advisor assigned. Then the student submits the semester fees and obtains the fee receipt and gets the signature of the Accounts Officer by obtaining the fee receipt. After this process, Dean Nominees acknowledges and confirms all the signatures and receipts and finally the forms are submitted in the administrative block. The student also receives one of the copies of the Yellow Form. As is evident, the student and faculty are hassled whilst completing the registration formalities and moreover, it leads to an added discomfort.

\section{Proposed System}

The web application system consists of five modules in total, namely Admin, Masters, Transactions, Reports and Utilities:

\section{Admin Module}

The administrative module is managed by the administrator. It is responsible for creating, managing, authenticating and authorizing different users and user types. Currently we have six user types i.e. Student, Head of Department, Registrar, Accounts Officer, Dean and Faculty. Under the Admin module the administrator can create and delete users and user types.

\section{Masters Module}

Masters Module manages database entries of all the master tables from the front-end. The Masters database is more or less permanent and is modified only at session start. The Masters Module currently manages these databasetables namely: category, college, country, course, course-category, credit/non-credit, department, designation, faculty, gender, qualification, quota, student, stream, Ug/Pg.

\section{Transactions Module}

Transactions Module facilitates the entries for Programme of Work and Student Registration from the front-end of the web application. Programme

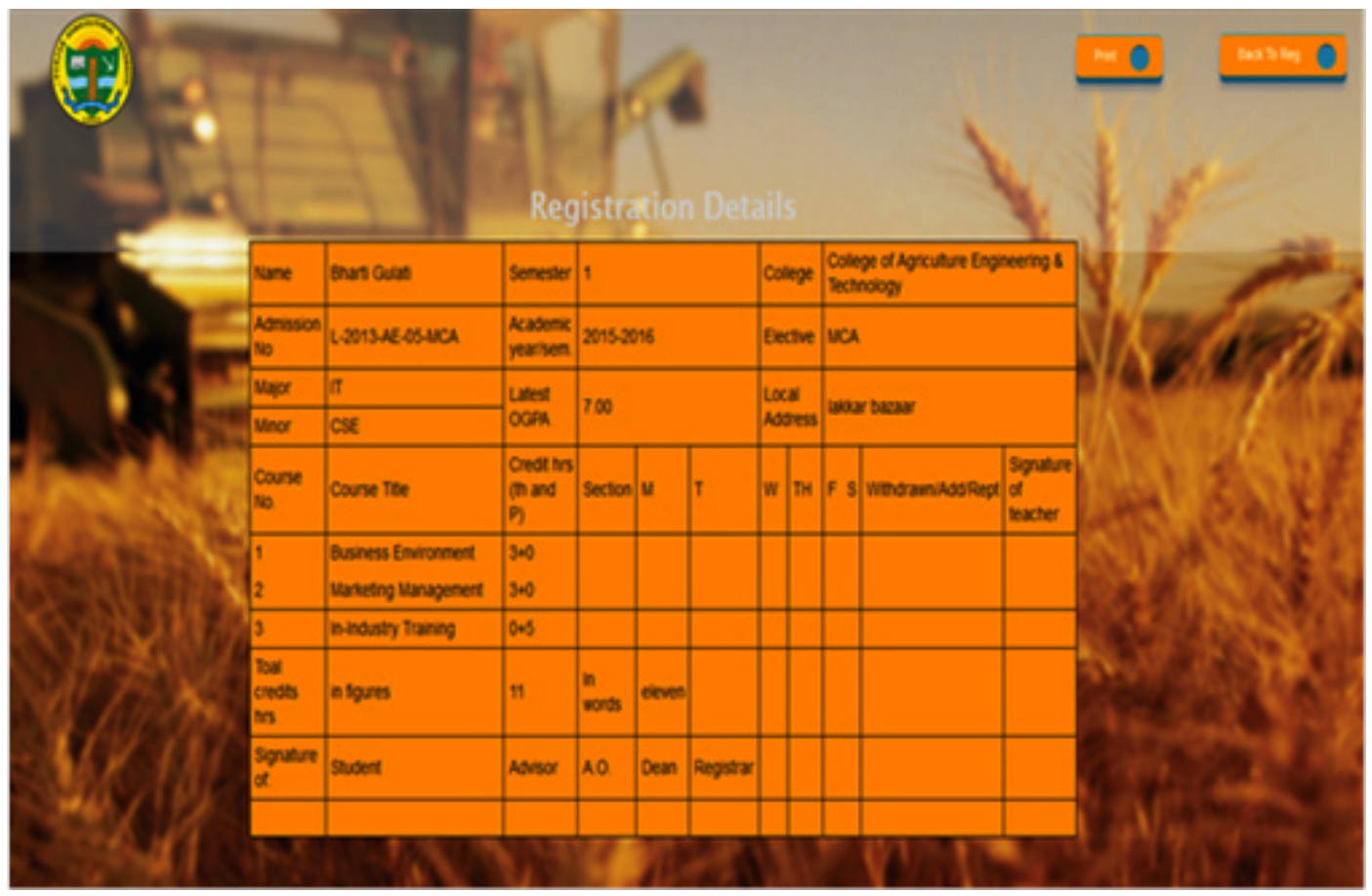

Fig. 2: Final Output after completing Registration Process 
of Work is created when a student is first enrolled with the college and Registration is done at the beginning of every semester. Programme of Work contains details of all the courses to be studied by the student during his/her degree. Registration caters to the courses a student has to study during the current semester. The List of Registration courses is obtained from the Programme of Work database.

\section{Reports Module}

Reports Module comprises of the final view of the entries made into the Admin, Masters and Transactions Modules. The information depicted inside the Reports Module is not visible to everyone in order to maintain data security, data integrity and consistency. The information is available according to the type of user logged into the system. This module contains reports for all the Admin, Masters and Transaction modules.

\section{Utilities}

Utilities menu contains two entries namely: Backup and Documentation. Backup offers options to Back up the database tables in sql format and can be saved in the root directory. As per need, the user can take back up of some or all tables at any time. Documentation contains the project documentation prepared which contains the detailed project system requirements, analysis and future scope of the system.

\section{System Development Life Cycle (SDLC) Analysis}

A detailed analysis report was prepared before designing the project. Initially the registration system was manual and every step in this system was performed manually which wasted time of all concerned.

\section{Feasibility Analysis}

An online registration system was developed where a student can register himself. The registration form has been designed to be user friendly and easy to fill and hence leads to saving of time and money as compared to multiple forms filled manually by the students. The software development team ascertained the technical feasibility of the project and concluded that the project can be undertaken with available technology and resources. Although implementation phase might require hardware additions but currently the project is technically feasible and should proceed further. The operational feasibility analysis acknowledged the acceptability of the provided solution to the problem. This analysis verified that the new system will be acceptable and adaptable to the new users. The economic feasibility study perceived that the project will produce long term gains for the institution. The cost benefit analysis proved that benefits of the proposed system undermine the costs involved, hence the system is worth implementing. The utility it provides to the students for completing the registration process and the provision it provides to the faculty for managing the database makes this project feasible to undertake.

\section{Design}

While designing the web application portal a three tier architecture for application development was followed. The presentation tier occupies the front end design of the application. It relates to every entity with which the user interacts. It accepts user inputs and actions, and then sends this information to the data tier through the application tier for further processing. The student course registration portal accepts input in the form of student's personal detail, student's registration details, faculty details etc. The second tier is application tier, it serves as an intermediary to the presentation tier and the data tier. It applies business logic to the input received from the presentation tier. The business logic is applied combining the information collected from upper and lower layers of the three tier architecture. When a student or a faculty member asks for a report to be retrieved, on the basis of this command data is fetched from the database and the required business logic is applied and finally a report is displayed to the user. The third and final tier is the data tier which is concerned with the design of the database to be used with the web application. It is concerned with maintaining central servers and its information. The data tier is kept independent of application servers and business logic to promote data security. The database is developed using MySQL keeping in mind the authenticity concerns of different users. All the necessary primary keys and access controls are depicted with utmost care.

In recent times, there has been an increase 
in use of Web enabled transaction processing online. Many organizations have adopted this technique for processing payments and selling products online, making travel reservations etc. Online Student Course Registration System is based on the Web enabled transaction processing model. The design flow of the web application starts with the student logging into the system and selecting the courses one wants to opt. Then the system verifies the data again with the database and sends it to the concerned advisors, dean and/or faculty. After the process is completed, a notification is sent to Advisor, Dean, Registrar and Student. There are in total 23 tables in the database for managing student data namely Category, Cnc, College, Country, Course, Crs_category, Department, Designation, Faculty, Gender, Pow, Qualification, Quota, Registration, Stream, Student, Stu_reg_ detail, Temp_course, Temp_reg, UG/PG, User, Usertypes and Validations. These tables further contain multiple columns for storing information. The database has been normalized which helps to maintain data integrity and prevents duplication of data. Initially before pursuing the project a system flow was prepared in order to answer the following questions:

- How will data flow In and Out of the system?

- How to maintain relationship between different data?

- what will be the different milestones data will travel from start to end?

To understand these questions and assess the requirements, pictorial representation was prepared which is given below in Figure 1: The front-end design of the web application is designed carefully for usage simplicity. There is a menu bar at top of the page for navigation and the user can scroll through different pages with its help. The final product of the registration system describes the student registration information in the format of the Yellow Form and is depicted below in Figure 2:

\section{Testing}

The compiled project was tested thoroughly using different usage environments like different browsers, Operating Systems, hardware components and different sets of data. The web application was found to be working conveniently. However, latest versions of the browser, Operating System and hardware components of the computer are recommended for best results.

\section{RESULTS AND DISCUSSION}

Development of Student registration system has greatly simplified previously complex tasks. Manual work is reduced to a large extent. The task of maintaining huge data can now be accomplished with a few button clicks. Previously, the system was hectic for both faculty and the students but now that it is accessible anywhere, registration process has been simplified. The database is maintained on a central server, so that it can be accessed anywhere inside the university by authorized personnel. Different departments can access the data whenever required. Also, the process being simple and easy to use any student or faculty with minimum computer knowledge can use the application easily. The user can print the final registration card and keep the information safe with him/her. Also, this detail can be saved as a PDF file anywhere on the computer.

Further, In future we would like to recommend certain valuable additions to the project. The most important is allowing students to submit the fees online. This can be achieved using the services of an online payment system portal. This addition would further reduce the student requiring, visiting the college premises physically. It would completely automate the given system. Also, a system to digitally sign the documents can also be implemented in future. This would enable the faculty and registrar to acknowledge the documents more securely and reduce the security risk to valuable personal information. Entering student grades at semester end is also recommended to be implemented in the future. Finally we can say that the Online Student Course Registration System for Student is ready for implementation and will surely provide the students and every entity associated with it a better experience. Although the project is complete and ready for implementation there is always room for improvement. As this system depends upon computer hardware, and with more and more student entries into the database there will be constant need to update server storage. 
To make the system more secure, In future digital acknowledgement signatures and SSL encryption can be implemented.

\section{CONCLUSION}

Implementing the Online Student Course Registration System, the registration procedure has been simplified. Previously student had to go door to door in order to get the documents acknowledged from the concerned officials whereas the currently developed system offers an efficient way to perform these operations. The students can access the registration portal online either from a computer or a smart phone, and fill the necessary information and submit it for further approval. This web application provides us with ease of access, user friendliness and transparency. On the other hand, from organizations viewpoint, it helps in maintaining transparency, data consistency, data accessibility and easy maintenance.

Implementation of the proposed system will reduce the workload of all those involved as the data can be now managed with proper authentication and authorizations instead of being hard copied and accessible to everyone. This system will largely save the precious time of Deans, Advisors and Accounts Officers, Instead of explicitly signing every document; they just have to acknowledge entries online with the click of a mouse. All the technologies i.e. PHP, Apache and MySQL used for current system design are open source and hence freely available for download. PHP provides a strong platform for creating the visual front-end of the web application and PHP combined with HTML provides a very flexible development environment. For the purpose of fulfilling Web Server requirements XAMPP was used, which is again Open Source and is supported across multiple platforms. In order to maintain visual consistency jQuery was used for simpler implementation of certain features. For constant testing, analysis and execution needs, Firefox and Google Chrome web-browsers were used. With a combination of all these technologies we were able to create a web application environment that is efficient and consistent enough.

The primary objective of our research and development was to automate student course registration procedure. It has been achieved successfully and the system is tested to be working efficiently. The student enters his/her information during the beginning of the semester, the system verifies the data entered, compares it to the previous semester entries in the database and forwards it to the concerned faculty. After getting the nod from the faculty and respective officials the registration form is submitted to the administrative staff of the college or university for further necessary action at their end.

Online application of the whole system helps easy access to the system anywhere. Physical presence of the student is not required. The time taken for process completion is now largely reduced. After registration the database is automatically updated at the end of process completion removing the hassle for department officials who had to enter the data manually. As the database is managed through MySQL, data duplication is eliminated and thereby reducing chances of error. Also data can be now be easily retrieved, edited and printed whenever required. Authentication based access proves to be more secure than manual system. The data is maintained on a central server and is distributed among different departments as per requirement and copies of this database are maintained on backup servers. Also, database access is authorized and cannot be viewed or edited by unauthorized personnel. So, this automated and computerized system is safe, fast and user friendly.

\section{ACKNOWLEDGEMENT}

The authors are indebted to the advisory members for their continuous guidance and monitoring and for providing the opportunity to work on a live project inside the university data centre. 


\section{REFERENCES}

1. Kaur, Harpreet, \& Grover, Dinesh. “Design and Development of Online Hospital Management Information System" International Journal of Computer Science Engineering and Information Technology Research 3.2 79-88 (2013).

2. Williams, Hugh E., and David Lane. "Web database applications with PHP and MySQL”. O’Reilly Media, Inc., 2004.

3. Meloni, Julie C. "Sams teach yourself PHP, MySQL and Apache all in one". Sams Publishing, 2012.

4. Ullman, Larry. "PHP advanced for the World Wide Web”. Peachpit Press, 2002.

5. Brinzarea, Bogdan, and Audra Hendrix. "Ajax and PHP: Building modern Web applications". Packt Publishing Ltd, 2009.

6. Thomas, D., White-Cinis, J., Lea, C., \& Buzzard, M. "PHP MySQL Website Programming: Problem-Design-Solution. Apress" (2003).

7. Glass, M. K., Le Scouarnec, Y., Naramore, E., Mailer, G., Stolz, J., \& Gerner, J. "Beginning PHP, Apache, MySQL Web Development”. John Wiley \& Sons (2004).

8. Capps, Stephen P. "Online service registration system and method." U.S. Patent No. 6,711,682. 23 Mar. 2004.

8. Shafie, E., Al-Ajlan, A., Aldrawiesh, K., Bajahzar, A., \& Al-Saawy, Y. (2011, January).
"Online registration system". In Advanced Computer Control (ICACC), 2011 3rd International Conference on (pp. 174-179).

9. Davis, Michele E., and Jon A. Phillips." Learning PHP \& MySQL: Step-by-Step Guide to Creating Database-Driven Web Sites”. O’Reilly Media, Inc., 2007.

10. Dembla, Pamila, Prashant Palvia, and Balaji Krishnan. "Understanding the adoption of web-enabled transaction processing by small businesses."Journal of Electronic Commerce Research 8.1 (2007): 1.

11. "Systems development Life Cycle"techtarget. com. Retrieved on April 15, 2016, from http://searchsoftwarequality.techtarget.com/ definition/systems-development-life-cycle.

12. "Software Development Process"wikipedia. com. Retrieved on April 17, 2016, from https://en.wikipedia.org/wiki/Software_ development_process

13. "Feasibility Study" wikipedia.com. Retrieved on April 9, 2016, from https://en.wikipedia. org/wiki/Feasibility_study

14. "Three Tier Architecture" techopedia.com Retrieved on April 20, 2016, from https:// www.techopedia.com/definition/24649/ three-tier-architecture

15. "PHP 5 Tutorials"w3schools.com. Retrieved on April 9, 2016, from http://www.w3schools. com/php/ 